\title{
RETRACTED ARTICLE: Proposal of a Performance Bound Estimation Process for Adaptive Transmissions in Land Mobile Satellite Channels
}

\author{
Daniele Tarchi
}

Published online: 7 February 2014

C Springer Science+Business Media New York 2014

This article, published in doi:10.1007/s11277-014-1645-y, has been retracted at the request of the author, because of a data entry error, which is fundamental to the study findings. As background, this was performance bound study based on the statistical output of a database of measures. The process involved the postprocessing of several variables and numerical data from different electronic sources aiming to extrapolate a statistical behaviour.

In proceeding to design a follow-up study to this work, Daniele Tarchi went back to the original source file to abstract new data. In doing this he identified a 'cut-and paste' error in which the source data from different set have been incorrectly assigned to specific statistical processing procedures. This led to a critical error that then got carried forward and a fundamental misclassification of the findings in the final study group. This error fundamentally affects the results, which now do not fully support the conclusions.

D. Tarchi $(\bowtie)$

Department of Electrical, Electronic and Information Engineering, University of Bologna, Bologna, Italy e-mail: daniele.tarchi@unibo.it 